\title{
Health-Related Optimism and Quality of Life among Diabetes Patients: the Moderating Role of Clinical Factors in a Nigerian Sample
}

\author{
Umukoro Omonigho Simon* \\ Department of Psychology, Faculty of the Social Sciences, Nigeria
}

*Corresponding author: Umukoro Omonigho Simon, Department of Psychology, Nigeria.

Received Date: October 23, 2018

Published Date: November 19, 2018

\begin{abstract}
This study examined severity of comorbidity and adherence to treatment as contextual moderators in the link between health-related optimism and quality of life among diabetic patients. The study adopted a cross-sectional survey design in which questionnaires were used for data collection from diabetic patients in two major hospitals within the Ibadan metropolis. A multistage sampling technique was employed in selecting a sample of 385 participants for the study. The sample consisted of 232 males and 153 females with a mean age of 64.69 years, and a standard deviation of 10.56. Results showed that the first model of treatment adherence and health related optimism explained $11.94 \%$ of the variance in quality of life among diabetes patients, while the interaction term accounted for a significant change in R2 by $3.45 \%$. The results implied that health related optimism positively predicted quality of life when treatment adherence was optimum $(\beta=2.0295 ; \mathrm{p}<.01)$; while there was no influence of health related optimism on quality of life when treatment adherence was poor $(\beta=.2126 ; p>.05)$. Similarly, the second model of comorbidity severity and health related optimism explained $9.30 \%$ of the variance in quality of life among diabetes patients; while the interaction term accounted for a significant change in R2 by $3.45 \%$. The result implied that health related optimism positively predicted quality of life when severity of comorbidity was high $(\beta=.6746 ; p<.01)$ or low $(\beta=3.6161 ; p<.01)$; however, a higher effect was recorded at the latter than the former. Implications of the results were highlighted and discussed.
\end{abstract}

Keywords: Diabetes; Severity of comorbidity; Adherence to treatment; Health-related optimism; Quality of life

\section{Background}

Nigeria, with a population of over 160 million people, is the most populous country in Africa and accounts for one sixth of Africa's population. The prevalence of diabetes in Nigeria has increased from $2.2 \%$ in 1997 to $5.0 \%$ in 2015 [1]. This rate of increasing prevalence of diabetes has been reported to affect the quality of life among patients in Nigeria [2]. Yet, insight into the association of combined bio-psychosocial variables and quality of life among diabetics is limited from a Nigerian perspective. Insight into these associations can facilitate a clearer understanding of quality of life in diabetes, which in turn can contribute to the design of optimally effective clinical interventions, while safeguarding a patient's quality of life.

Diabetes is a common disease that affects all age groups [3]. Diabetes is sometimes called 'the silent killer' because is a chronic metabolic disorder and a major health and physical problem that is increasing significantly particularly in developing countries. The main goal of diabetic treatment is not only to relieve physical symptoms of the disease, but also to improve the patients' quality of life [4]. Quality of life is an important issue, because if ignored, it can lead to frustration, lack of motivation and decrease in social activities. Quality of life definition is difficult because it is a broad and complex concept. It is recognized as having a sense of satisfaction and happiness and is defined as individuals' perception of life [5]. Tang WL [6] defined the quality of life as those features which are valuable for patient and are factors of good feeling or good perception. These features are in line with physical, emotional and intellectual functioning development, so that persons with these abilities can maintain valuable activities in life.

Quality of life can be theorized to play an important role in treatment outcomes for diabetes from the patient's point of view. 
Diabetes has many daily self-management demands of a patient: They must make countless decisions every day in an effort to maintain their glycaemic control. Patients often feel frustrated, overwhelmed or afraid by this never-ending daily self-care. Despite these demotivating factors, patients need to maintain glycaemic control in order to reduce the risk for cardiovascular complications. Short or long-term cardiovascular complications can make the disease even more burdensome [7]. Indeed, quality of life has been found to be impaired in patients with diabetes [8]; however, in line with the tenets of positive psychology, health related optimism may be a vital antecedent towards enhancing patients' quality of life [9]

Health related optimism refers to a disposition or tendency to look on the more favorable side of health related events or conditions and to expect the most favorable health outcomes. Optimism, as conceived by Scheier MF, et al. [10] is related to ego strength and internal control. Positive states of mind have long been associated with health and successful coping while depression, despair, and hopelessness have been linked to capitulation, illness, and even death [11]. Systematic reviews on a number of studies have reported an apparent connection between health outcomes and states of hope, optimism or pessimism [12]. However, criticisms of the impact of health related optimism in fostering quality of life among patients with long term health complications have appeared in the form of theoretical arguments [13] that highlight the critical role of various clinical factors that may moderate the relationship between health optimism and quality of life.

In this vein this study focuses on degree of comorbid illnesses and adherence to treatment as contextual moderators in the link between health-related optimism and quality of life among diabetic patients. Comorbidity is the presence of one or more additional diseases or disorders co-occurring with (that is, concomitant or concurrent with) a primary disease or disorder. Patients with diabetes may not only have diabetes-related comorbidity but also have non diabetes-related comorbidity, such as depression and musculoskeletal diseases [14]. Adherence to treatment is defined as the degree to which a patient correctly follows medical advice. Most commonly, it refers to medication or drug compliance, but it can also apply to other situations such as medical device use, selfcare, self-directed exercises, or therapy sessions [15]. Healthcare professionals have clamoured for a continuous need to evaluate the associations of comorbidities as well as treatment adherence in persons with diabetes and examine how they influence treatment outcomes.

To guide the direction of this study, the following hypotheses will be tested.

Hi1: Treatment Adherence will moderate the influence of health-related optimism on quality of life among diabetic patients.

Hi2: Degree of comorbid illnesses will moderate the influence of health-related optimism on quality of life among diabetic patients.

\section{Theoretical Framework}

The Health Belief Model (HBM) [16] is the only model which has been developed specifically to explain health behaviour and has generated the most research in this area. It is relevant to behaviours that are under an individual's control. The model was originally conceived in order to predict preventive health behaviours but with later adaptations has also been used to predict the behaviour of people with acute and chronic illnesses. The value-expectancy approach in the context of health-related matters was translated as: (a) the desire to avoid illness, or if ill, to get well; and (b) the belief that a health behaviour will prevent or ameliorate illness. The likelihood of someone taking a health action is seen to be determined by the individual's perceptions about his/her susceptibility to an illness and the perceived severity of its consequences.

Taken together these are said to constitute the perceived threat or risk of the illness which provides the energy or force to act. The behavioural outcome, on the other hand, is influenced by an evaluation of the required action in terms of its efficacy in reducing the threat (perceived benefits) weighed against the perceived costs of or barriers to undertaking the behaviour. Thus, the following four perceptions serve as the main constructs of the model: perceived seriousness, perceived susceptibility, perceived benefits, and perceived barriers. Each of these perceptions, individually or in combination, can be used to explain health behavior. More recently, other constructs have been added to the HBM; thus, the model has been expanded to include cues to action, motivating factors, and self-efficacy. These major constructs of perception are modified by other variables, such as culture, education level, past experiences, skill, and motivation, to name a few.

\section{Methods}

\section{Design and participants}

This study adopts a cross-sectional survey design in which questionnaires were used for data collection from diabetic patients in two major hospitals within the Ibadan metropolis. A sample of about 385 patients participated in the study. This sample size was calculated using the Z-test based on the average reports of diabetic cases in Ibadan. A multistage sampling technique was employed in selecting participants for the study. The first stage was achieved through convenience sampling in selecting the hospitals that make up the research setting. Factors considered for the selection included proximity to the researcher, size of hospital, popularity of hospital, consent of participation from hospital management etc. the second stage involved purposive sampling technique employed in selecting the study participants. This was achieved by approaching every diabetic patient that came into clinic for checkup or consultations. The patients were approached during their regular check-up visits to the hospitals. Consent of participants was however obtained. The sample consisted of 232 males and 153 females with a mean age of 64.69 years, and a standard deviation of 10.56 .

\section{Measures}

Health related optimism: Health related optimism was assessed using a revised Life Orientation Test (LOT-R) [17]. The measure includes 10 statements rated on a five point response format ranging from 1 (not at all) to 4 (very much so). Items 3, 7 , and 9 are reverse scored (or scored separately as a pessimism measure). Items 2, 5, 6, and 8 are fillers and should not be scored. Scoring is kept continuous. Means of the positively and negatively 
worded items are calculated separately to yield optimism scores and pessimism scores. The authors obtained a Cronbach alpha of .81 for the scale.

Severity of comorbid illness: Severity of comorbid illness was assessed using a self-report measure developed in line with the Diabetes Complications Severity Index (DCSI). The DCSI uniquely incorporates a wide range of diabetes complications and is a useful tool for predicting baseline severity of disease in the diabetic population. The DCSI was developed to model the severity of diabetes complications at any one point in time. The severity index includes the following seven categories of complications: cardiovascular disease, nephropathy, retinopathy, peripheral vascular disease, stroke, neuropathy, and metabolic. Items were tailored in line with these categories and presented in a Likert response format. Participants were to identify diseases within each category that are comorbidities to their current diabetic state and then indicate the level of severity on a five point likert scale for each disease. A Cronbach alpha of 0.81 was established via an initial pilot study.

Adherence to treatment: Adherence to treatment was measured using the Morisky Medication Adherence Scale by Morisky et al. [18]. This self-report scale consists of 7 items answered with a yes or no and 1 item with a 5-point Likert scale ranging from $1=$ Never to $5=$ Very Often. The scores of the MMAS- 8 range from 0 to 8 . A score below 6 indicates low adherence, a score between $6<8$ medium adherence and a score of 8 high adherence. The 8-item MMAS has established a second level criterion related validity, following comparisons with pharmacy fill rates. The original MMAS-8 study had achieved internal consistency reliability $\alpha=0.83$ [18]. The current study showed satisfactory Cronbach's alpha of 0.753 . All items had item to total correlations greater than 0.30. Prior published reports on validating the MMAS-8 have reported internal consistency reliability with Cronbach's alpha between 0.61 to 0.675 in patients with diabetes [19].

Quality of life: Quality of Life was measured using The Quality of Life Inventory (QOLI) by Frisch [20]. The QOLI is a 16-item self-report instrument measuring perceived importance of and satisfaction in 16 life domains, such as health, work, community, and friendships. For each domain, respondents rate the importance of that area on a 5-point scale ranging from 'very unsatisfied=1' to 'very satisfied=5'. The QOLI has demonstrated high test-retest reliability and high internal consistency across clinical and nonclinical samples $(\alpha=.82)$, as well as good criterion validity [21]. The QOLI has shown good convergent validity with other measures of subjective well-being, and correlates significantly and negatively with measures of psychopathology, depression, and anxiety [21].

\section{Analytical procedure}

For analyses of data, the study employed a moderated multiple regression using PROCESS macro [22] which analyzes direct and conditional effects of moderators in a model. It entails the addition of an interaction term (predictor x moderator) in a linear multiple regression to determine if the effect of the predictor variable on the response variable varies at different values of the moderator variable.

\section{Hypothesis Testing}

\section{Hypothesis one}

The first hypothesis stated that treatment adherence will moderate the influence of health-related optimism on quality of life among diabetic patients. Results from Table 1a show that the interaction term produced significant effect $(\beta=2.2421 ; \mathrm{p}<.01)$ and accounted for a significant increase in R2 by $3.45 \%$. Overall, the model explained $11.94 \%$ of the variance in quality of life among diabetes patients. The results imply that treatment adherence significantly moderated the influence of health related optimism on quality of life among diabetic patients. Further results in Table $1 \mathrm{~b}$ shows the conditional influence of health related optimism at values of treatment adherence. Outcomes in Table $1 \mathrm{~b}$ show that health related optimism positively predicts quality of life when treatment adherence is optimum $(\beta=2.0295 ; \mathrm{p}<.01)$; while there is no influence of health related optimism on quality of life when treatment adherence is poor $(\beta=.2126$; $p>.05)$. This implies that optimum treatment adherence is a necessary condition for a positive impact of health related optimism on quality of life among diabetes patients. The hypothesis stated is therefore accepted.

Table 1a: Model showing moderation effects of treatment adherence on the influence of health related optimism on quality of life.

\begin{tabular}{|c|c|c|c|c|c|c|}
\hline & \multirow{2}{*}{ B } & \multirow{2}{*}{ SE } & \multirow{2}{*}{$\mathbf{T}$} & \multirow{2}{*}{$\mathbf{P}$} & \multicolumn{2}{|c|}{$95 \% \mathrm{CI}$} \\
\hline & & & & & LLCI & ULCI \\
\hline Constant & 28.5627 & 20.4192 & 1.3988 & 0.1627 & 11.5868 & 68.7121 \\
\hline Health Related Optimism & 2.4547 & 0.98 & 2.50448 & 0.0127 & 0.5278 & 4.3816 \\
\hline Treatment Adherence & 36.5464 & 9.3391 & 3.9133 & 0.0001 & 18.1832 & 54.9096 \\
\hline Interaction Term & 2.2421 & 0.5829 & -3.8465 & 0.0001 & 3.3882 & 1.096 \\
\hline
\end{tabular}

\section{DV: Quality of Life}

Model Summary: $R^{2}=.1194, F_{(3,381)}=8.5559 ; p<.01$

$\Delta R^{2}$ due to interaction: $\Delta R^{2}=.0345, F_{(1,381)}=14.7958 ; p<.01$

Table 1b: Conditional effects of health related optimism at values of treatment adherence.

\begin{tabular}{|c|c|c|c|c|c|c|}
\hline Treatment Adherence & Effect & S.E & T & P & LLCI & ULCI \\
\hline Poor & 0.2126 & 0.4519 & 0.4704 & 0.6383 & 0.676 & 1.1012 \\
\hline Optimum & 2.0295 & 0.3572 & 5.6811 & 0 & 2.7319 & 1.3271 \\
\hline
\end{tabular}

DV: Quality of Life 


\section{Hypothesis two}

The second hypothesis stated that severity of comorbidity will moderate the influence of health-related optimism on quality of life among diabetic patients. Results from Table 2a show that the interaction term of comorbidity severity and health-related optimism produced significant effect $(\beta=2.9414 ; \mathrm{p}<.01)$ and accounted for a significant increase in R2 by $4.27 \%$. Overall, the model explained $9.30 \%$ of the variance in quality of life among diabetes patients. The results imply that severity of comorbidity significantly moderated the influence of health related optimism on quality of life among diabetic patients. Further results in Table $2 \mathrm{~b}$ shows the conditional influence of health related optimism at values of comorbidity severity. Outcomes in Table $2 b$ show that health related optimism positively predicts quality of life when severity of comorbidity is high $(\beta=.6746 ; p<.01)$ or low $(\beta=3.6161$; $\mathrm{p}<.01$ ); however, a higher effect is recorded at the latter than the former. This implies that health related optimism is has a more positive impact on quality of life among diabetes patients when severity of comorbidity is low. The hypothesis stated is therefore accepted.

Table 2a: Model showing moderation effects of comorbidity severity on the influence of health related optimism on quality of life.

\begin{tabular}{|c|c|c|c|c|c|c|}
\hline & \multirow{2}{*}{ B } & \multirow{2}{*}{ SE } & \multirow{2}{*}{$\mathbf{T}$} & \multirow{2}{*}{$\mathbf{P}$} & \multicolumn{2}{|c|}{$95 \% \mathrm{CI}$} \\
\hline & & & & & LLCI & ULCI \\
\hline Constant & 93.0666 & 4.9767 & 18.7005 & 0 & 83.2814 & 102.8518 \\
\hline Health Related Optimism & 0.6746 & 0.3086 & 2.186 & 0.0294 & 1.2815 & 0.0678 \\
\hline Severity of Comorbidity & 47.8528 & 11.1521 & 4.209 & 0 & 25.9255 & 69.7801 \\
\hline Interaction Term & 2.9414 & 0.6946 & 42,346 & 0 & 4.3072 & 1.5757 \\
\hline
\end{tabular}

DV: Quality of Life

Model Summary: $R^{2}=.0930, F_{(3,381)}=13.020 ; p<.01$

$\Delta R 2$ due to interaction: $\Delta R^{2}=.0427, F_{(1,381)}=17.9319 ; p<.01$

Table $2 \mathbf{b}$ : Conditional effects of health related optimism at values of comorbidity severity.

\begin{tabular}{|c|c|c|c|c|c|c|}
\hline Severity of Comorbidity & Effect & S.E & T & P & LLCI & ULCI \\
\hline High & 0.6746 & 0.3086 & 2.186 & 0.0294 & 1.2815 & 0.0678 \\
\hline Low & 3.6161 & 0.6223 & 5.8109 & 0 & 4.8396 & 2.3925 \\
\hline
\end{tabular}

\section{Discussion}

Findings of this study show that positive thinking and positive thoughts lead to a better quality of life in diabetic patients. This is justified by the fact that optimism enables people to think honestly about themselves and to overcome the worries and stresses that are present in everyday life. A person with an optimistic orientation toward the future evaluates stressful situations with a positive view and has a good calculation of his abilities to deal with problems. Optimistic individuals in the face of stressful events show selfreliance and they are more likely to consider positive outcomes about the future and use more problem-oriented coping strategies to deal with problems. As a result, optimism seems to play an important role in adapting people to stressful situations.

Given the increasing incidence of diabetes and its associated health challenges, a positive state of mind among patients diagnosed with diabetes is thus likely to enhance their quality of life while undergoing treatment options. Previous studies have provided similar results in showing that positive beliefs have a positive relationship with various dimensions of quality of life. For instance, results by Kepka, et al. [23] affirm that optimism reduced the negative effects of anxiety; with an accompanying significant relationship between optimism and quality of life. Hassan SM, et al. [24] found that patients who reported high levels of optimism often adopted realistic coping mechanisms in leading fulfilling lives during health challenges. Brydon, et al. [25] also concluded that optimism has a protective role against the inflammatory effects of psychological stress; while Vilhena E, et al. [26] found that dispositional optimism is more likely to play a significant role in quality of life pathway among patients with chronic ailments.

The initial hypothesis of the current study however sought to examine the moderating role of treatment adherence in the relationship between health-related optimism and quality of life among diabetes patients. The results showed that optimum treatment adherence is a necessary condition for a positive impact of health related optimism on quality of life among diabetes patients. This is because at optimum levels of treatment adherence, health related optimism positively predicts quality of life; however at low levels of treatment adherence, health related optimism has no influence on quality of life. The plausibility of the results might stem from the mirror effect between optimism and treatment adherence. This implies that optimism levels of treatment outcomes may shape patterns of treatment adherence; and patterns of treatment adherence may reflect levels of optimism about treatment outcomes. This may therefore provide a contextual basis for the moderating role of treatment adherence in the initial model of the study.

One way in which patients will be better able to manage their illnesses is by adhering to their medication and treatment regimens. Many patients, especially patients with a chronic illness, experience difficulties in following treatment recommendations [27]. According to the WHO, adherence to long-term therapy for chronic illnesses averages only $50 \%$. As a result of poor adherence, patients do not receive optimal benefit from their drug therapy. Suboptimal treatment can lead to increased use of health care services (acute 
care and hospitalizations), reduction in patient's quality of life, and increased health care costs $[28,29]$. The reports of World Health Organization have emphasized that "increasing the effectiveness of adherence interventions may have a far greater impact on the quality of health among patient populations than any improvement in specific medical treatments". In line with the moderating influence of treatment adherence in this study, Martínez, et al. [30] found that influence of knowledge and attitude on patients' quality of life domains was moderated by their treatment adherence behaviour.

The second hypothesis examined the moderating role of comorbidity severity in the relationship between health-related optimism and quality of life among diabetes patients. Outcomes of the study showed that health related optimism had a more positive impact on quality of life among diabetes patients with less severe comorbidities than more severe comorbidities. The results therefore confirm the positive moderating role of comorbidity severity at both levels of severity, albeit differences in its level of potency at both levels. In justifying the results, it has been established that the comorbidities associated with diabetes are its major challenges among patients; therefore comorbidity severity levels are implicated in patients' expectations and perceptions of treatment outcomes. Expectations in form of optimism levels and perceived quality of life may thus be affected in relation to the challenging nature of the comorbidities experienced by the patient.

In lay terms, a diabetic patient with less severe comorbidities may express optimism towards treatment outcomes and consequently experience good quality of life while the realities of more severe comorbidities may dampen optimism levels and perceived quality of life. Comorbidities can have profound effects on patients' ability to manage their self-care. Depression and arthritis impair patients' functioning and pose significant barriers to lifestyle changes and regimen adherence [31]. Conditions such as emphysema and chronic low back pain can have a more debilitating impact on patients' health status than diabetes per se [32] and are among the most important determinants of diabetic patients' functioning and quality of life [33]. In addition, disabling conditions such as advanced heart failure and dementia may make standard diabetes self-care goals impossible to reach. Even when comorbid chronic conditions do not directly limit patients' ability to selfmanage their diabetes, these conditions can serve as competing demands which limit treatment optimism and perceived wellbeing [34].

\section{Conclusion}

In sum, clinicians and health systems seeking to improve diabetes management cannot avoid addressing the ways in which treatment adherence and comorbidities affect diabetes care. Many health systems in Nigeria are still poorly designed to support effective diabetes management [27], let alone grapple with the challenges that arise when patients are struggling with multiple concurrent conditions. Thus, improving diabetes management requires a more holistic, patient-centered approach which should not only focus on providing treatment options for diabetes patients, but should incorporate psycho-educative interventions aimed at enhancing levels of optimism and treatment adherence. Management and coping with associated comorbidities of diabetes should also take center-stage in such psycho-educative interventions.

\section{Acknowledgement}

None.

\section{Conflict of Interest}

No Conflict of Interest.

\section{References}

1. Oputa RN. Chinenye S (2015) Diabetes in Nigeria - a translational medicine approach. African Journal of Diabetes Medicine, 23(1): 7-10.

2. Adeniyi AF, Ogwumike OO, Oguntola DA \& Adeleye JO (2015) Interrelationship among physical activity, quality of life, clinical and sociodemographic characteristics in a sample of Nigerian patients with type 2 diabetes. African Journal of Physiotherapy and Rehabilitation Sciences 7(1\&2): 12-18.

3. American Diabetes Association (2015) Diabetes basics.

4. Asif M (2014) The prevention and control the type- 2 diabetes by changing lifestyle and dietary pattern. J Educ Health Promot 3: 1.

5. Snock FG (2000) Quality of life: A closer look at measuring patients wellbeing. Diabetes spectrum 13(4): 24.

6. Tang WL, Wang YM, Du WM, Cheng NN, Chen BY (2006) Assessment of quality of life and relevant factors in elderly diabetic's patients in the Shanghai community. Pharmacoepidemiol Drug Saf 15(2): 123-130.

7. Bradley C, Plowright R, Stewart J, Valentine J, Witthaus E (2007) The Diabetes treatment satisfaction questionnaire change version (DTSQc) evaluated in insulin glargine trials shows greater responsiveness to improvements than the original DTSQ. Health Qual Life Outcomes 5: 57.

8. Brod M, Hammer M, Christensen T, Lessard S, Bushnell DM (2009) Understanding and assessing the impact of treatment in diabetes: the Treatment-Related Impact Measures for Diabetes and Devices (TRIMDiabetes and TRIM-Diabetes Device). Health Qual Life Outcomes 9(7): 83.

9. Seligman MEP (2008) Positive Health. Applied psychology: An international review 57: 3-18.

10. Scheier MF, Carver CS (1987) Dispositional optimism and physical wellbeing: The influence of generalized outcome expectancies on health. J Pers 55(2): 169-210.

11. Arnau RC, Rosen DH, Finch FJ, Rhudy JL, Fortunato VJ (2007) Longitudinal effects of hope on depression and anxiety: a latent variable analysis. J Pers 75(1): 43-63.

12. Schiavon CC, Marchetti E, Gurgel LG, Busnello FM, Reppolld CT (2017) Optimism and hope in chronic disease: a systematic review. Front Psychol 7: 2022.

13. Rai R, Jongenelis M, Pettigrew S, Jackson B, Newton RU (2017) Identifying modifiable factors associated with health optimism in older adults. Aging Ment Health 22: 1-9.

14. Egede L, Zheng D, Simpson K (2002) Comorbid depression is associated with increased health care use and expenditures in individuals with diabetes. Diabetes Care 25(3): 464-470.

15. Elliott RA, Marriott JL (2009) Standardised assessment of patients' capacity to manage medications: A systematic review of published instruments. BMC Geriatr 9: 27.

16. Rosenstock LM, Strecher VJ, Becker MH (1988) Social learning theory and the health belief model. Health Educ Q 15(2): 175-183.

17. Scheier MF, Carver CS, Bridges MW (1994) Distinguishing optimism from neuroticism (and trait anxiety, self-mastery, and self-esteem): A reevaluation of the Life Orientation Test. J Pers Soc Psychol 67(6): 10631078. 
18. Morisky DE, Ang A, Krousel-Wood M, Ward HJ (2008) Predictive validity of a medication adherence measure in an outpatient setting. J Clin Hypertens (Greenwich) 10(5): 348-354.

19. Sakthong P, Chabunthom R, Charoenvisuthiwongs R (2009). Psychometric properties of the Thai version of the 8-item Morisky medication adherence scale in patients with type 2 diabetes. Ann Pharmacother 43(5): 950-957.

20. Frisch MB (1994) Quality of Life Inventory. Minneapolis, USA.

21. Frisch MB, Cornell J, Villanueva M, Retzlaff PJ (1992) Clinical validation of the Quality of Life Inventory. A measure of life satisfaction for use in treatment planning and outcome assessment. Psychological Assessment 4(1): 92-101

22. Hayes AF (2013) Methodology in the social sciences. Introduction to mediation, moderation, and conditional process analysis: A regressionbased approach. Guilford Press, New York, US.

23. Kepka S, Baumann C, Anota A, Buron G, Spitz E, et al. (2013) The relationship between traits optimism and anxiety and health-related quality of life in patients hospitalized for chronic diseases: Data from the SATISQOL study. Health Qual Life Outcomes 11: 134.

24. Hassan SM (2002) The relationship between optimism and coping strategies in university students. Quarterly J Fundament Mental Health 4(15): 86-98.

25. Brydon L, Walker C, Wawrzyniak AJ, Chart H, Steptoe A (2009) Dispositional optimism and stress-induced changes in immunity and negative mood. Brain Behav Immun 23(6): 810-816.

26. Vilhena E, Pais-Ribeiro J, Silva I, Pedro L, Meneses RF, et al. (2014). Optimism on quality of life in Portuguese chronic patients: moderator/ mediator? Rev Assoc Med Bras (1992) 60(4): 373-380.
27. Awodele 0, Osuolale JA (2015) Medication adherence in type 2 diabetes patients: study of patients in alimosho general hospital, igando, lagos, Nigeria. Afr Health Sci 15(2): 513-522.

28. Lau DT, Nau DP (2004) Oral antihyperglycemic medication nonadherence and subsequent hospitalization among individuals with type 2 diabetes. Diabetes Care 27(9): 2149-2153.

29. Sokol MC, McGuigan KA, Verbrugge RR, Epstein RS (2005) Impact of medication adherence on hospitalization risk and healthcare cost. Med Care 43(6): 521-530.

30. Martínez YV, Prado-Aguilar CA, Rascón-Pacheco RA, Valdivia-Martínez JJ (2008) Quality of life associated with treatment adherence in patients with type 2 diabetes: A cross-sectional study. BMC Health Ser Res 8(1): 164.

31. Wolff JL, Starfield B, Anderson G (2002) Prevalence, expenditures, and complications of multiple chronic conditions in the elderly. Arch Intern Med 162(20): 2269-2276.

32. Maddigan SL, Feeny DH, Johnson JA (2005) Health-related quality of life deficits associated with diabetes and comorbidities in a Canadian National Population Health Survey. Qual Life Res 14(5): 1311-1320.

33. Jha AK, Perlin JB, Kizer KW, Dudley RA (2003) Effect of the transformation of the Veterans Affairs Health Care System on the quality of care. N Engl J Med 348(22): 2218-2227.

34. Wilson C, Gilliland S, Cullen T, Moore K, Roubideaux Y, Valdez L, Vanderwagen W, Acton K (2005) Diabetes outcomes in the Indian health system during the era of the Special Diabetes Program for Indians and the Government Performance and Results Act. Am J Public Health 95(9): 1518-1522. 